\title{
Polysaccharides Derived from Saposhnikovia divaricata May Suppress Breast Cancer Through Activating Macrophages
}

This article was published in the following Dove Press journal: OncoTargets and Therapy

\author{
Jingxian Ding ${ }^{1 *}$ \\ Yonghong Guo ${ }^{2, *}$ \\ Xiaoliu Jiang' \\ Qingge $\mathrm{Li}^{1}$ \\ Kai Li' \\ Min Liu' \\ Wenbing $\mathrm{Fu}^{\prime}$ \\ Yali $\mathrm{CaO}^{3}$
}

'Department of Radiation Oncology, The Breast Cancer Institute, The Third Hospital of Nanchang, Nanchang, Jiangxi Province 330025, China; ${ }^{2}$ Department of Radiation Oncology, The Fourth Affiliated Hospital of Nanchang University, Nanchang, Jiangxi Province 330003, China; ${ }^{3}$ Department of Breast Surgery, The Breast Cancer Institute, The Third Hospital of Nanchang, Nanchang, Jiangxi Province 330025, China

*These authors contributed equally to this work
Correspondence: Jingxian Ding; Yali Cao Email jingxianding10@fudan.edu.cn; caoyali@medmail.com.cn
Background: Saposhnikovia divaricata (SD) has been used in traditional Chinese medicine to treat pain, inflammation, and arthritis. Recently, it has been reported that SD extract may inhibit tumor growth, but the mechanism involved is elusive. The aim of this study was to investigate the anti-tumor activity of polysaccharides derived from SD in breast cancer and the underlying mechanisms.

Materials and Methods: Polysaccharides isolated from SD were analyzed using Fourier transform infrared (FT-IR) spectroscopy and gas chromatography-mass spectroscopy (GCMS). Their effects on cell growth of U937, MCF-7, and MDA-MB-231, and tumor growth in a mouse MDA-MB 231 xenograft model were examined. Their role in U937 activation, MCF-7, and MDA-MB 231 cytokine release profiles were also tested.

Results: In vitro studies showed that SD polysaccharides (SDPs) promoted U937 cell growth dose-dependently, with no obvious effect on growth of breast cancer cell lines MCF-7 and MDA-MB-231. SDP also showed an antagonistic effect against the growth inhibition of U937 by the culture supernatants of MCF-7 and MDA-MB-231, and reversed the polarization status of U937. Treatment of SCID mice bearing MDA-MB-231-derived xenograft tumors with SDP significantly reduced tumor growth. At all tested concentrations, no obvious toxic side-effects were recorded.

Discussion: We tentatively concluded that SDPs potently promote the growth of U937 and activate it to inhibit the tumor growth of SCID mice bearing MDA-MB-231-derived xenograft tumors indirectly, with no obvious growth inhibition effects on MCF-7 and MDA-MB -231 in vitro. Our finding indicated that SDP could be a potential anticancer agent for breast cancer.

Keywords: breast cancer, Saposhnikovia divaricata, polysaccharides, immuno-regulation, macrophage

\section{Introduction}

Breast cancer is one of the most common malignant tumors in women and the main cause of cancer related death among women worldwide, including China. ${ }^{1,2}$ Despite the great achievements made in breast cancer research and treatment, measures for hormone receptor negative and human epithelial growth factor receptor-2 (HER2) negative, the so-called triple negative breast cancer (TNBC), are still deficient. Over the last years, the intricate balance of interactions between cancer cells and their microenvironment (TME) has emerged as a crucial regulator of tumor progression and metastasis. Tumor-associated macrophages (TAMs) are the main constituents of 
the TME in various solid tumors including breast cancer. ${ }^{3-6}$ Macrophages differentiate from the mononuclear phagocytic lineage, and their differentiation is dependent on the cytokines in the microenvironment. The activation of monocyte mainly involves two pathways. The classical pathway (M1) is activated by cytokines like interferon- $\gamma$, bacterial lipopolysaccharide, and tumor necrosis factor alpha. While the alternative pathway (M2) is triggered by cytokines, like IL4, IL-13, and macrophage colony-stimulating factor (M-CSF, also known as CSF1). ${ }^{7-9}$ CSF1 is a secreted cytokine which activates hematopoietic stem cells to polarize into macrophages and is involved in the proliferation, differentiation and survival of monocytes, macrophages, and bone marrow progenitor cells. $^{9-12}$ The M1 pathway mediates the defense against bacterial pathogens, while the M2 pathway may show a "pro-tumorigenic" effect by producing high levels of IL-10, TGF- $\beta$, and low levels of IL-12. TAMs normally have M2 phenotype, and seem to have a pro-tumorigenic effect. ${ }^{8,13}$ TAMs are recruited to tumors in response to diverse tumorderived chemotactic factors. Our previous study showed that CSF1 expression level was much higher in estrogen receptor negative breast cancer cell line MDA-MB-231 and MDAMB-468 cultural supernatants compared to that of estrogen receptor positive cell line MCF-7. Our finding supported that CSF1 regulated macrophage differentiation, migration, and survival. $^{11}$

Saposhnikovia divaricata (SD) is a traditional Chinese herb, namely "Fangfeng", which has been intensively used to treat rheumatism, arthralgia, headaches, stroke, fever, cold, etc. ${ }^{14}$ Polysaccharides are chains of sugar units that form in configurations from tens to thousands of units long, which comprise the carbohydrate storage units for plants. Polysaccharides derived from SD, namely SD Polysaccharides (SDP), have proved to be of various activities, such as analgesic, anti-convulsant, anti-inflammatory, anticoagulant, antipyretic activities, and anti-cancer. ${ }^{15-17}$ There is accumulating evidence suggesting the important role of immune-suppression induced by tumor cells in the progression of tumor aggressiveness. Immuno-suppression is also shown to be a major barrier in overcoming cancer. ${ }^{18-22}$ Previous studies showed that the SDP had effects on immuno-regulation, anti-oxidant, and anti-tumor activity in a tumor xenograft mouse. ${ }^{22,23}$ However, the structure of SDP was still unclear, which limited our understanding of the structural activity relationship of SDP. Recent reports on SDP showed that their major composition was arabinose (Ara), galactose (Gal), glucose (Glu), and galacturonic acid (GalA). ${ }^{24}$ Nevertheless, it must be noted that the structure of
SDP extracted from the same origin is usually varied, which could be explained by different preparation and purification processes, or even because of the different analytical methods. $^{24}$

In this study, the crude SDP were extracted and isolated from Saposhnikovia divaricata by Ultrasonicassisted extraction. Their main sugar compositions were analyzed with Fourier Transform Infrared (FT-IR) spectroscopy and Gas Chromatography-mass spectroscopy (GCMS). Furthermore, the pharmacological activities of SDP were investigated on the U937 macrophage cell line, MCF-7 and MDA-MB-231 breast cancer cell lines.

\section{Materials and Methods \\ Ethics Statement}

All animal protocols were supervised and approved by the Animal Care Committee of Shanghai Institutes for Biological Sciences, Chinese Academy of Sciences (permit no. IBS18-0806). Mice were maintained in laminar flow rooms with stable temperature and humidity conditions. All mice were sacrificed by cervical dislocation to minimize suffering, which was conducted according to the Laboratory Animal Guidelines for Ethical Review of Animal Welfare.

\section{Cell Lines and Reagents}

MDA-MB-231 and MCF-7 breast cancer cell lines were maintained in our institute, which were originally obtained from the American Type Culture Collection (ATCC). The U937 human promonocytic cell line was a gift from Professor Duan Ma (Key Laboratory of Molecular Medicine, Ministry of Education, Shanghai Medical College, Fudan University), which also originated from the ATCC. SD was purchased from the Pharmacy Department of our hospital, whose growth place was Inner Mongolia, China. Trypsin from bovine pancreas, dimethyl sulfoxide (DMSO), rhamnose (Rha), arabinose (Ara), xylose (Xyl), mannose (Man), glucose (Glc), and galactose (Gal) were purchased from Sigma (St. Louis, MO, USA). RPMI 1640 supplemented with $10 \%$ fetal bovine serum (FBS) and 100 units/mL penicillin/streptomycin was obtained from GIBCO (Invitrogen, Carlsbad, CA, USA). All other reagents used in this study were of analytical grade.

\section{The Extraction of SDP}

SDP were extracted by ultrasound-assisted extraction technology as previously reported with slight modifications. ${ }^{25}$ 
Briefly oven-dried SD (500 g) was smashed and pulverized to pass through a 60 -mesh sieve, $5 \mathrm{~L}$ deionized distilled water was added to a flat-bottomed flask. The solidliquid ratio of $1: 10 \mathrm{~g} / \mathrm{mL}$ was determined as optimal conditions. The extraction was performed with an ultrasonic machine (Kun Shan Ultrasound Instrument Co., Jiangsu, China). The extraction temperature, time, ultrasonic frequency, and power were $60^{\circ} \mathrm{C}, 20$ minutes, $80 \mathrm{KHz}$, and $220 \mathrm{~W}$, respectively. The extraction was performed thrice, and the extract of each run was centrifuged to remove debris fragments of the polysaccharides extracts. The supernatants were collected after filtration and concentrated with a rotary evaporator and precipitated with four volumes of $95 \%$ ethanol $(\mathrm{V} / \mathrm{V})$ for 24 hours at $4^{\circ} \mathrm{C}$. The supernatants were incubated at $37^{\circ} \mathrm{C}$ for 2 hours after adding $20 \mathrm{mg}$ trypsin. The protein digestion was stopped by sitting the container into the boiling water bath for 20 minutes. The denatured proteins were removed by centrifuge at $10,000 \mathrm{~g} / \mathrm{min}$, for 15 minutes, and the SDP were collected with filtration and dried with a vacuum dryer.

\section{Analysis of Sugar Composition}

The analysis of sugar composition was performed as previously reported. ${ }^{26}$ Briefly, the SDP were hydrolyzed with $2 \mathrm{M}$ trifluoroacetic acid (TFA) at $120^{\circ} \mathrm{C}$ for 2 hours in order to completely hydrolyze polysaccharides. After the removal of TFA under nitrogen gas, the hydrolyzed monosaccharide compositions were determined using a GC-MS system (7890B system, Agilent Technologies, Palo Alto, CA, USA) fitted with an Agilent $\mathrm{J} \& \mathrm{~W}$ capillary column $(30 \mathrm{~m} \times 250 \mu \mathrm{m} \times 0.25$ $\mu \mathrm{m})$ and a flame-ionization detector (FID). Mass spectra of monosaccharide composition of the SDP were compared with the standards neutral sugar included Rha, Ara, Gal, Glu, Xyl, Man.

\section{Fourier-Transform Infrared (FT-IR) Analysis of Polysaccharides}

The crude SDP were identified by using a $\mathrm{KBr}$ presseddisk method on a FT-IR Spectrometer in the frequency ranging from 4000 to $400 \mathrm{~cm}^{-1}$. Briefly, the dried SDP were ground with $\mathrm{KBr}$ powder and then pressed into $1 \mathrm{~mm}$ pellets for measurement. FT-IR spectra were recorded on a Fourier Transform Infrared Spectrometer (Nicolet iS10, Thermo Fisher Scientific) fitted with a DTGS detector at absorbance mode from 4000 to $400 \mathrm{~cm}^{-1}$.

\section{Cell Proliferation Assay}

The SDP were dissolved in DMSO in a concentration of $200 \mathrm{mg} / \mathrm{mL}$ stocked solution. The stocked solution was diluted by phosphate buffered saline (PBS) in a suitable concentration for use. To study the effects of SDP on cell proliferation, cell proliferation assay was assessed using CCK-8 (Dojindo Laboratories, Kumamoto, Japan). Briefly, 5000 cells/well were plated in a 96-well plate in triplicate for each group. After the cells had stabilized overnight, triplicate samples of cells were treated with culture medium containing various concentrations $(0,12.5,25,50$, $100,200,400$, and $800 \mu \mathrm{g} / \mathrm{mL}$ ) of SDP alone, or in combination with breast cancer conditioned culture medium. After treatment for 48 hours, $10 \mu \mathrm{L} /$ well of CCK-8 was added to each well, and the cells were incubated at $37^{\circ} \mathrm{C}$ for another 2 hours. Optical density was measured at $460 \mathrm{~nm}$ using a microplate reader. Three readings were obtained, with wells without cells used as the blank control.

\section{Reverse Transcription-PCR Analysis}

The total RNA was extracted from $1 \times 10^{6}$ cells using TRIzol (Invitrogen) RNA isolation method according to the manufacturer's instruction. Briefly, $1 \mu \mathrm{g}$ of total RNA was reverse transcribed using PrimeScript ${ }^{\circledR}$ RT-PCR Kit (Takara Bio, Dalian, China) primed with oligo (dT). After synthesis of the complementary DNA (cDNA), $1 \mu \mathrm{L}$ of cDNA was subjected to PCR amplification using targeted gene primers. CD163 expression was measured by quantitative real-time PCR (qRT-PCR) technique. Quantitative PCR was carried out using the SYBR ${ }^{\circledR}$-Green PCR Master Mix Kit (Takara Bio). Primer sequences are detailed in Table 1. GAPDH expression was the inner control.

\section{Measurement of Cytokine Secretion}

In determining the effects of SDP on the expression profile of tumor associated cytokines, we measured the cytokine secretion profile by Bio-Plex ${ }^{\circledR}$ cytokine assay following the manufacturer's instructions (Bio-Rad Laboratories, Inc., San Diego, CA, USA). Briefly, MCF-7 or MDA-MB-231 breast cancer cells $\left(1 \times 10^{6}\right.$ cells $\left./ \mathrm{mL}\right)$ were incubated for 24 hours at $37^{\circ} \mathrm{C}$ in a $5 \% \mathrm{CO}_{2}$-humidified atmosphere. The medium was replaced by fresh culture medium containing $400 \mu \mathrm{g} / \mathrm{mL}$ of SDP or not, then incubated for another 48 hours. Afterwards, the cultural supernatants were collected and stored at $-80^{\circ} \mathrm{C}$ after centrifugation to remove 
Table I Characteristics of PCR primer sets and products

\begin{tabular}{|l|l|l|}
\hline Gene & $\begin{array}{l}\text { Size of PCR Products } \\
\text { (bp) }\end{array}$ & $\begin{array}{l}\text { Primer Sequences } \\
\left(\mathbf{5}^{\prime} \rightarrow \mathbf{3}^{\prime}\right)\end{array}$ \\
\hline GAPDH & 200 & $\begin{array}{l}\text { F: acccagaagactgtggatgg } \\
\text { R: tctagacggcaggtcaggtc }\end{array}$ \\
\hline CDI63 & 146 & $\begin{array}{l}\text { F: cgagttaacgccagtaagg } \\
\text { R: gaacatgtcacgccagc }\end{array}$ \\
\hline
\end{tabular}

cells and their debris until testing. Cytokine levels were measured using the Bio-Plex Pro human cytokine 27Plex premixed immunoassay kit according to manufacturer's instructions. Standard curves for each cytokine were established by Bio-Plex Manager ${ }^{\mathrm{TM}}$ using the reference concentrations provided in the kit. Detailed procedures of the Bio-Plex ${ }^{\circledR}$ Suspension Array System have been reported previously. ${ }^{11}$

\section{Breast Cancer Xenografts}

To explore the effects of SDP on tumor growth in vivo, non-obese diabetic/severe combined immunodeficient (NOD/SCID) 8-week-old female mice were used to make breast cancer xenografts. Briefly, $5 \times 10^{6}$ MDA-MB-231 or MCF-7 cells suspended in $100 \mu \mathrm{L}$ PBS were injected orthotopically into the left mammary fat pad according to standard injection procedures, as previously reported (30). The tumors were allowed to grow for up to 14 days without intervention. The tumor-bearing mice were randomly divided into an untreated group as the control and three treatment groups $(200,400$, and $800 \mu \mathrm{g} / \mathrm{g}$ SDP), with five mice per group. Treatment groups received one gavage of indicated concentrations of SDP once a day for 35 consecutive days. The control group received the same volume of the vehicle (PBS). Tumor diameters and body weights of mice in each group were measured with digital calipers and recorded each week. The tumor volume was calculated as $\left(\right.$ width $^{2} \times$ length) $/ 2$. Tumor growth curves were plotted based on the calculated tumor volume. After the last gavage, the mice in all groups were fasted for 24 hours and then were weighed and sacrificed by cervical dislocation and necropsied. The tumors were then excised and weighed, and their sizes were measured using vernier calipers, then paraffin-embedded for further study.

\section{Immunohistochemistry}

For immunohistochemical analysis, paraffin-embedded xenograft tumor blocks of MDA-MB-231 and MCF-7 were cut into $5 \mu \mathrm{m}$ serial sections. CD163 antibody (clone 10D6, Novocastra, UK) used for immunostaining was titered to find the optimal concentration (1:100). Sections were counterstained with hematoxylin for the identification of nuclei, and immunohistochemistry was performed following standard procedures. Detection was performed using the DAKO Envision system. Images were captured under a microscope with a CCD camera.

\section{Statistical Analysis}

Data were analyzed from three independent experiments using GraphPad Prism, and were recorded as mean \pm SE. A comparison among different groups was performed using multivariate one-way ANOVA analyses. $P<0.05$ was considered with statistical significance.

\section{Results}

\section{Extraction and Monosaccharide Composition of SDP}

Previous reports showed that ultrasonic-assisted extraction allowed higher penetration of solvent into the raw plant materials was a rapid and effective extraction technique that used ultrasonic waves to generate a cavitation in the solvent. ${ }^{25}$ Compared with conventional extraction methods, Ultrasonic-assisted extraction was more efficient, environmentally friendly, and easier to use. It could greatly decrease the extraction time. The extraction temperature of the ultrasound-assisted extraction was lower than the temperature of the conventional solvent extraction. The yield of polysaccharides was higher than conventional solvent extraction. However, there was no significant difference of polysaccharides chemical compositions among different extraction. The higher efficiency of ultrasound-assisted extraction was because of cells broken by ultrasound and polysaccharides dissolved more easily in the solvent. The extraction parameters of ultrasound-assisted extraction might also affect the yield and pharmacological activities of polysaccharides. The determined extraction temperature, time, ultrasonic frequency, and power were $60^{\circ} \mathrm{C}, 20$ minutes, $80 \mathrm{KHz}$ and $220 \mathrm{~W}$, respectively in this study. The yield of SDP was $6.45 \%$ by weight under these parameters.

Because of its high resolution, sensitivity, specificity, and easy sample treatment, the GC-MS has become a popular method to analyze the monosaccharide composition. Colorimetric analyses showed no uronic acid was detected in the prepared extraction samples. The monosaccharide composition of the isolated SDP were 


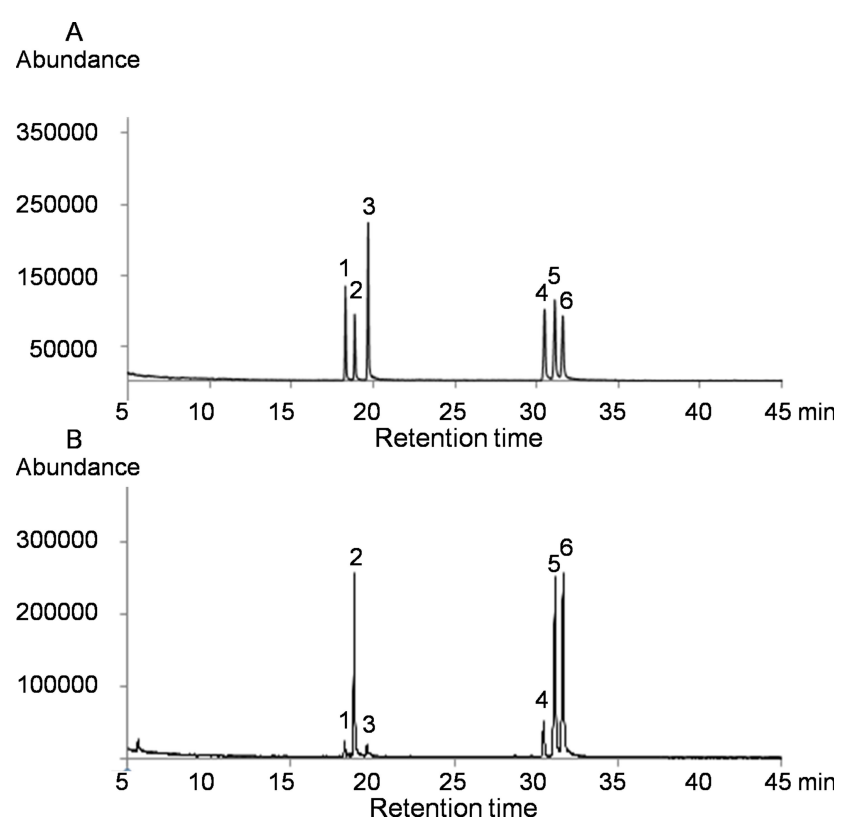

Figure I The Gas chromatography-mass spectroscopy (GC- MS) profiles of completely hydrolyzed standards (A) and polysaccharides isolated from Saposhnikoviadivaricata (B). The major monosaccharide contents of the polysaccharides isolated from Saposhnikoviadivaricata were Arabinose (Ara), Glucose (Glu), and Galactose (Gal), with a molar ratio of approximately I:I:I.

Abbreviations: Rha, rhamnose; Ara, arabinose; Xyl, xylose; Man, mannose; Glu, glucose; Gal, galactose.

tested by GC-MS. Monosaccharide standards including Rha, Ara, Gal, Glu, Xyl, and Man were used to calibrate the retention time. The GC-MS chromatogram of monosaccharide standards is shown in Figure 1A. Monosaccharide components of SDP were determined by GC-MS analysis, and Figure 1B shows that SDPs were mainly composed of Ara, Glu, and Gal with a molar ratio of approximately 1:1:1. Our results are in accordance with the previous studies. ${ }^{22}$

FT-IR analysis was applied to further identify the fundamental groups present in SDP structure. FT-IR spectrometric characterization of SDP is shown in Figure 2, the FT-IR spectra showed mainly $\beta$-pyranoid-type glycosidic linkages.

\section{The Effects of SDP on Cell Proliferation}

The effects of SDP on cell proliferation were evaluated on U937, MCF-7, and MDA-MB-231 cells. SDP significantly stimulated the proliferation of U937 cells in a concentrationdependent manner, and concentrations of SDP up to $800 \mu \mathrm{g} /$ $\mathrm{mL}$ did not exhibit obvious cytotoxic effects on breast cancer cell lines in vitro (Figure 3A-C). These results suggested that SDP might not show direct cytotoxic effects on cancer cells even at the concentrations up to $800 \mu \mathrm{g} / \mathrm{mL}$.

\section{The SDP Antagonized the Immunosuppression by Breast Cancer Cells on U937}

Accumulating evidence showed that polysaccharides might activate antitumor immune responses. Our previous study showed that breast cancer cell could induced U937 differentiate into M2 tumor associated macrophage, which exhibited immunosuppression and pro-tumor effects. ${ }^{27}$ Combinations of $400 \mu \mathrm{g} / \mathrm{mL}$ non-cytotoxic dosage of SDP with MCF-7 or MDA-MB-231 breast cancer cell culture supernatants were tested to see if they could produce antagonistic anti-proliferative effects on U937. The SDP exhibited antagonistic effects on cell proliferation of U937, as shown in Figure 4A. Moreover, SDP could antagonize the immunosuppression by MCF-7 and MDAMB-231 breast cells culture supernatants on U937 macrophages polarization as confirmed by RT-PCR (Figure 4B).

In determining the effects of SDP on cytokine secretion profile of breast cancer cells, MCF-7 and MDA-MB-231 were incubated with a $400 \mu \mathrm{g} / \mathrm{mL}$ non-cytotoxic dosage of SDP for 48 hours, respectively, and the cytokine secretion profile was analyzed by Bio-Plex Pro Human Cytokine 27plex Assay. Among the cytokines quantified, CSF1 concentration was much higher in MDA-MB-231 culture supernatants compared with that of MCF-7, and SDP reduced the CSF1 level in MDA-MB-231 cultural supernatants (Figure 5).

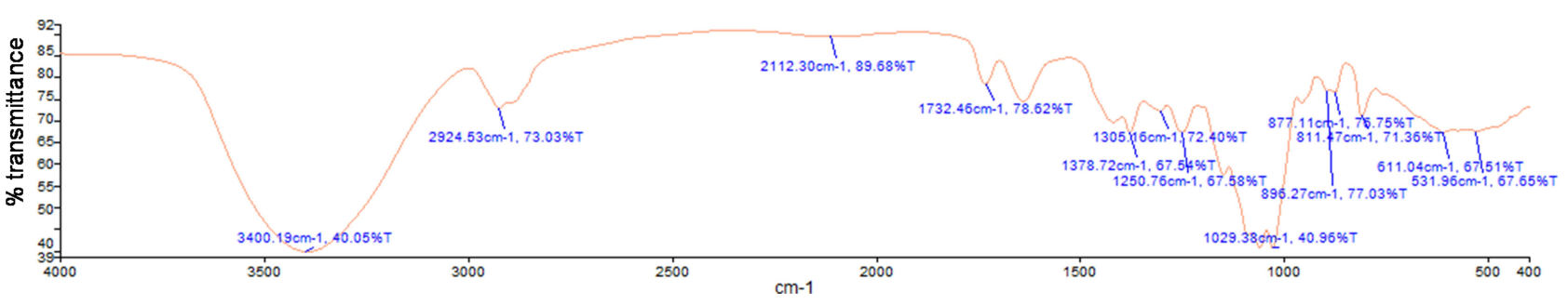

Figure 2 Fourier transform infrared (FT-IR) spectra of the polysaccharides isolated from Saposhnikovia divaricata were recorded at room temperature. FT-IR spectra were recorded using a Nicolet Magna-IR 550 spectrophotometer in the $4000-400 \mathrm{~cm}^{-1}$ region using $\mathrm{KBr}$ tables. Wave numbers (cm ${ }^{-1}$ ). 

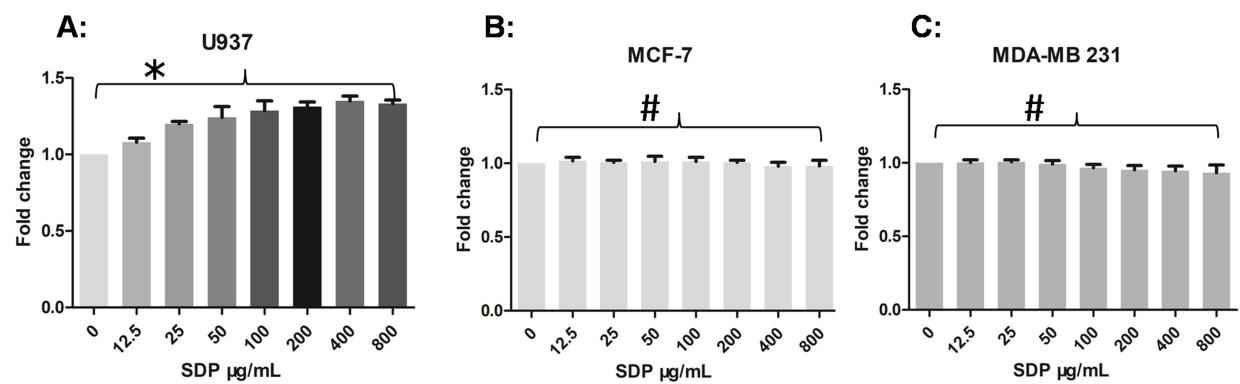

Figure 3 The proliferation effects of polysaccharides isolated from Saposhnikovia divaricata on U937 (A), MCF-7 (B), and MDA-MB-23I (C). SDP significantly stimulated the proliferation of U937 cells in a concentration dependent manner, while concentrations of these polysaccharides up to $800 \mu \mathrm{g} / \mathrm{mL}$ did not exhibit obvious cytotoxic effects on breast cancer cell lines in vitro. Error bars indicate standard deviation of the means. Data are exhibited as mean \pm SE of three independent results. * Indicates a statistical significance compared with the control group $(P<0.05)$, while ${ }^{*}$ indicates no statistical significance $(P>0.05)$.
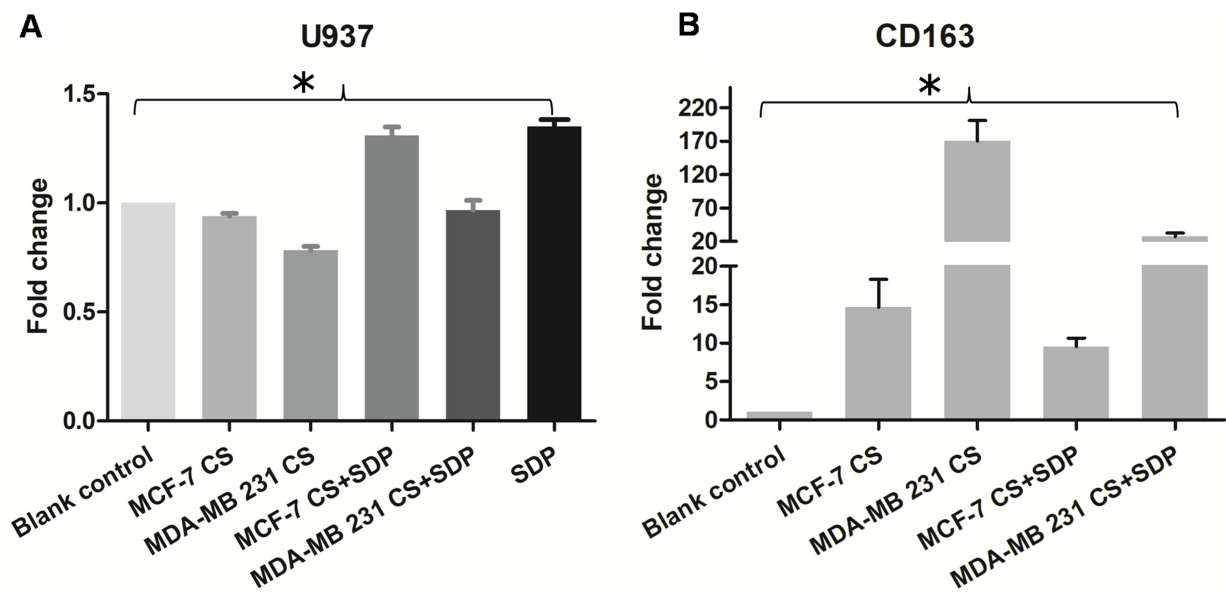

Figure 4 The effects of SDP on immunosuppression by breast cancer cells. (A) The SDP antagonized the immunosuppression by breast cancer cells on U937 after coculture with MCF-7 or MDA-MB-23I breast cancer cell cultural supernatants (CS). (B) The CDI63 mRNA expression level was increased in U937 cells after co-culture with MCF-7 or MDA-MB-23I breast cancer cell CS, while SDP attenuated the effect. * Indicates a statistical significance compared with the control group $(P<0.05)$.

\section{The SDP Suppressed Tumor Growth Dose-Dependently in MDA-MB-23 I Breast Cancer Xenografts}

In order to study the pharmacological activity of SDP in vivo, 8-week-old female NOD/SCID mice mammary fat pads were injected with $5 \times 10^{6}$ cells suspended in 100 $\mu \mathrm{L}$ PBS orthotopically. Two weeks later, the tumor-bearing mice were randomly divided into an untreated group as the control and three treatment groups (200, 400, and $800 \mu \mathrm{g} / \mathrm{g}$ SDP). The weak tumorigenic breast cancer cell line MCF7 was also used as a control. Tumor diameters and body weights of mice in each group were measured with digital calipers and recorded each week. The tumor volume was computed as (width ${ }^{2} \times$ length)/2. SDP suppressed tumor growth in a dose-dependent manner (Figure 6A). Tumor growth curves were also plotted (Figure 6B). Both the results of our in vitro and in vivo studies indicated that
SDP played an important role in suppressing breast cancer by activating macrophages.

\section{The SDP Antagonized TAMs Recruitment by Breast Cancer Cells}

Immunohistochemical analysis showed that the prevalence of CD163 positive macrophage was decreased in paraffinembedded xenograft tumors of MDA-MB-231 after SDP treatment. The prevalence of CD163 positive macrophage in MCF-7 xenograft tumors was very few. As the figures indicating in Figure 7, the SDP antagonized TAMs recruitment by breast cancer cells in human breast cancer xenografts mice.

\section{Discussion}

Accumulating studies have elucidated the important role of tumor immunosuppression in the progression of tumor aggressiveness. ${ }^{3,-7,-18-20}$ Immunosuppression is also 


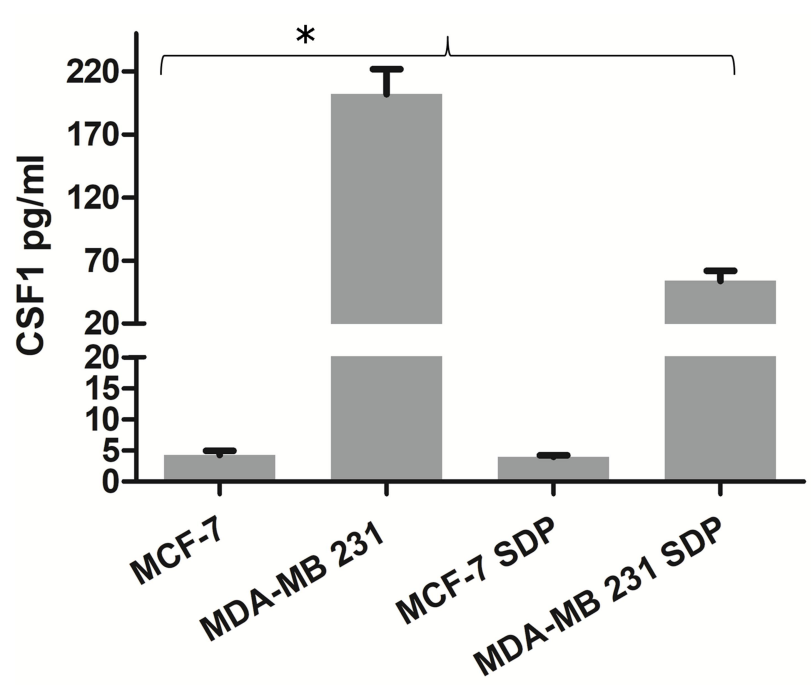

Figure 5 The effects of SDP on the cytokine secretion profile of MCF-7 and MDAMB-23I. The SDP reduces the concentration of CSFI in breast cancer cell lines culture supernatants. * Indicates a statistical significance compared with the control group $(P<0.05)$.

a major obstacle to conquering cancer. $^{28,29}$ The tumor macrophage plays an important role in cancer progression. $\mathrm{SD}$ is a perennial herb, and the dry root of this plant is known as Fangfeng, which has been widely applied in traditional Chinese medicine. This herb exhibits antiinflammatory, anti-arthritic, anti-tumor, and immunological activities. It is reported that the active ingredients of SD extract include chromones, polyacetylenes, coumarins, and polysaccharides, depending upon different methods of extraction. ${ }^{16,-30-39}$ Dong et $\mathrm{al}^{22}$ first reported the structural profile of SDPs and their antagonistic effects against the immunosuppression by the cultural supernatants of melanoma cells on RAW264.7 macrophages.
In this study, we found that SDP promoted U937 cell growth dose-dependently, while there was no obvious effect on growth of breast cancer cell lines MCF-7 and MDA-MB-231 in the tested concentration in vitro. SDP also showed an antagonistic effect against the growth inhibition of U937 by the culture supernatants of MCF-7 and MDA-MB-231, and reversed the polarization status of U937. Our finding also indicated that treatment of SCID mice bearing MDA-MB-231 derived xenograft tumors with SDP significantly reduced tumor growth. At all tested concentrations, no obvious toxic sideeffects were recorded. The pharmacological activity study indicated that SDPs showed an antagonistic effect against immunosuppression by breast cancer cells on macrophages. The SDP antagonized TAMs recruitment by breast cancer cells in human breast cancer xenografts mice. These properties of SDPs add to the potential use in cancer therapy other than its traditional usage in traditional Chinese medicine. We tentatively concluded that SDP potently promote the growth of U937 and activate it to inhibit the tumor growth of SCID mice bearing MDA-MB-231 derived xenograft tumors indirectly, with no obvious growth inhibition effects on MCF-7 and MDA-MB-231 in vitro.

Our finding indicates that SDP could be a potential anticancer agent for breast cancer. In conclusion, this study is the first to clarify SDP suppressing breast cancer on the basis of activating macrophages to regulate the host immunity. Further research is wanted to identify the suitable dosage and safety of SDP on human beings.

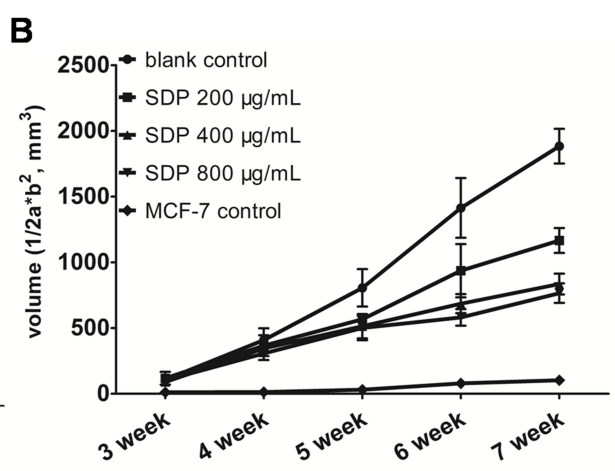

Figure 6 The effects of SDP on tumor growth in MDA-MB-23। breast cancer xenografts. MDA-MB-23। tumor-bearing mice were randomly divided into an untreated group as the control and three treatment groups (200,400, and $800 \mu \mathrm{g} / \mathrm{g}$ SDP), and the weak tumorigenic breast cancer cell line MCF-7 was also used as a control, with five mice per group. Tumor diameters and body weights of the mice in each group were measured with digital calipers and recorded each week. The tumor volume was computed as (width ${ }^{2} \times$ length) $/ 2$ the tumor-bearing mice. The SDP suppressed tumor growth in both dose-dependent and time-dependent manners, the tumor volume (A) and tumor

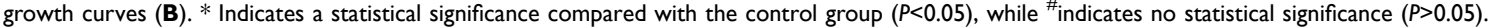


A

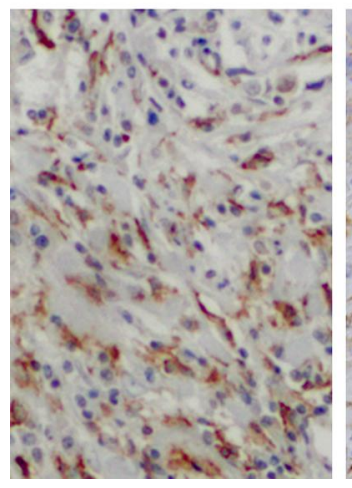

B

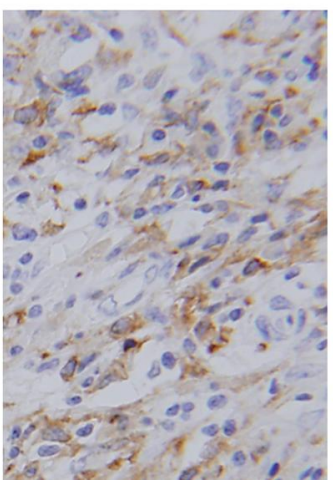

C

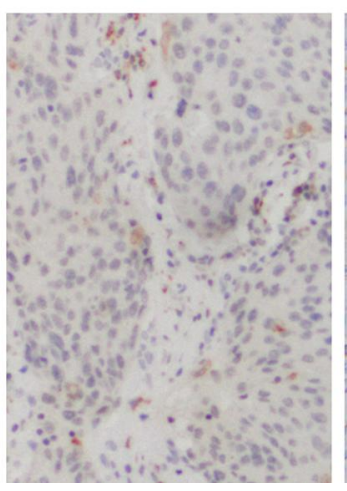

D

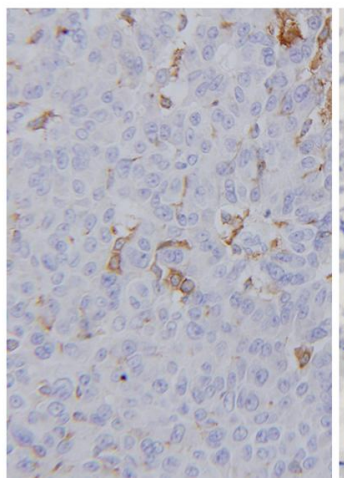

$E$

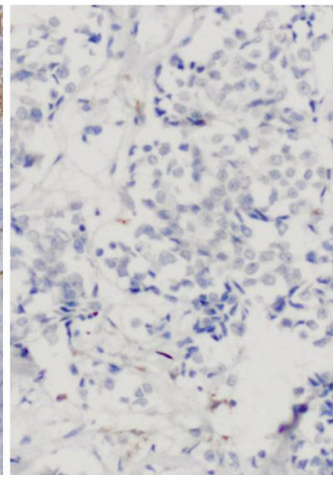

Figure 7 The SDP antagonized TAMs recruitment by breast cancer cells in human breast cancer xenografts mice. The prevalence of CDI63 positive macrophage among SDP treated and control mice through immunohistochemical analysis. As the figures indicates, the SDP antagonized TAMs recruitment by breast cancer cells in human breast cancer xenografts mice. (A) MDA-MB-23I blank control; (B) $200 \mu \mathrm{g} / \mathrm{g}$ SDP; (C) $400 \mu \mathrm{g} / \mathrm{g}$ SDP; (D) $800 \mu \mathrm{g} / \mathrm{g}$ SDP; and (E) MCF-7 control.

\section{Funding}

The study was sponsored by the Natural Science Foundation of Jiangxi province (No. 20171BAB205057). We wish to thank Dr. Wen in Jiangxi University of Traditional Chinese Medicine for performing the Fourier Transform Infrared (FT-IR) spectroscopy and Gas Chromatography-mass spectroscopy (GC-MS) analysis.

\section{Disclosure}

The authors report no conflicts of interest for this work. Jingxian Ding and Yonghong Guo contributed equally to this study.

\section{References}

1. Li H, Zheng RS, Zhang SW, et al. Incidence and mortality of female breast cancer in China, 2014. Zhonghua Zhong Liu Za Zhi. 2018;40 (3): $166-171$

2. Siegel RL, Miller KD, Jemal A. Cancer statistics, 2020. CA Cancer J Clin. 2020;70(1):7-30. doi:10.3322/caac.21590

3. Mantovani A, Sica A. Macrophages, innate immunity and cancer: balance, tolerance, and diversity. Curr Opin Immunol. 2010;22 (2):231-237. doi:10.1016/j.coi.2010.01.009

4. De Palma M, Biziato D, Petrova TV. Microenvironmental regulation of tumour angiogenesis. Nat Rev Cancer. 2017;17(8):457-474.

5. Sanchez-Gonzalez I, Bobien A, Molnar C, et al. miR-149 suppresses breast cancer metastasis by blocking paracrine interactions with macrophages. Cancer Res. 2020;80(6):1330-1341. doi:10.1158/ 0008-5472.CAN-19-1934

6. Obeid E, Nanda R, Fu YX, et al. The role of tumor-associated macrophages in breast cancer progression (review). Int $J$ Oncol. 2013;43(1):5-12. doi:10.3892/ijo.2013.1938

7. Gordon S, Martinez FO. Alternative activation of macrophages: mechanism and functions. Immunity. 2010;32(5):593-604. doi:10.1016/j.immuni.2010.05.007

8. Wynn TA, Chawla A, Pollard JW. Macrophage biology in development, homeostasis and disease. Nature. 2013;496(7446):445-455. doi:10.1038/nature 12034
9. Svensson J, Jenmalm MC, Matussek A, et al. Macrophages at the fetal-maternal interface express markers of alternative activation and are induced by M-CSF and IL-10. J Immunol. 2011;187 (7):3671-3682. doi:10.4049/jimmunol.1100130

10. Stanley ER, Berg KL, Einstein DB, et al. Biology and action of colony-stimulating factor-1. Mol Reprod Dev. 1997;46(1):4-10.

11. Ding J, Guo C, Hu P, et al. CSF1 is involved in breast cancer progression through inducing monocyte differentiation and homing. Int J Oncol. 2016;49(5):2064-2074. doi:10.3892/ijo.2016.3680

12. Stanley ER, Berg KL, Einstein DB, et al. The biology and action of colony stimulating factor-1. Stem Cells. 1994;12(Suppl 1):15-24, 25.

13. Geissmann F, Manz MG, Jung S, et al. Development of monocytes, macrophages, and dendritic cells. Science. 2010;327(5966):656-661. doi:10.1126/science.1178331

14. Kreiner J, Pang E, Lenon GB, et al. Saposhnikoviae divaricata: a phytochemical, pharmacological, and pharmacokinetic review. Chin J Nat Med. 2017;15(4):255-264.

15. Okuyama E, Hasegawa T, Matsushita T, et al. Analgesic components of saposhnikovia root (Saposhnikovia divaricata). Chem Pharm Bull (Tokyo). 2001;49(2):154-160. doi:10.1248/cpb.49.154

16. Ci B, Wang W, Ni Y. Inhibitory effect of Saposhnikovia divaricate polysaccharide on fibroblast-like synoviocytes from rheumatoid arthritis rat in vitro. Pak J Pharm Sci. 2018;31(6(Special)):2791-2798.

17. Yu X, Niu Y, Zheng J, et al. Radix Saposhnikovia extract suppresses mouse allergic contact dermatitis by regulating dendritic-cellactivated Th1 cells. Phytomedicine. 2015;22(13):1150-1158. doi:10.1016/j.phymed.2015.09.002

18. Schreiber RD, Old LJ, Smyth MJ. Cancer immunoediting: integrating immunity's roles in cancer suppression and promotion. Science. 2011;331(6024):1565-1570. doi:10.1126/science.1203486

19. Gross S, Walden P. Immunosuppressive mechanisms in human tumors: why we still cannot cure cancer. Immunol Lett. 2008;116 (1):7-14. doi:10.1016/j.imlet.2007.11.012

20. Torphy RJ, Schulick RD, Zhu Y. Newly emerging immune checkpoints: promises for future cancer therapy. Int J Mol Sci. 2017;18 (12):2642. doi:10.3390/ijms 18122642

21. Eckhardt BL, Francis PA, Parker BS, et al. Strategies for the discovery and development of therapies for metastatic breast cancer. Nat Rev Drug Discov. 2012;11(6):479-497.

22. Dong CX, Liu L, Wang CY, et al. Structural characterization of polysaccharides from Saposhnikovia divaricata and their antagonistic effects against the immunosuppression by the culture supernatants of melanoma cells on RAW264.7 macrophages. Int J Biol Macromol. 2018;113:748-756. doi:10.1016/j.ijbiomac.2018.03.022 
23. Meng Y, Yi L, Chen L, et al. Purification, structure characterization and antioxidant activity of polysaccharides from Saposhnikovia divaricata. Chin J Nat Med. 2019;17(10):792-800.

24. Wu F, Zhou C, Zhou D, et al. Structure characterization of a novel polysaccharide from Hericium erinaceus fruiting bodies and its immunomodulatory activities. Food Funct. 2018;9(1):294-306. doi:10.1039/C7FO01389B

25. Wang J, Lu HD, Muhammad U, et al. Ultrasound-assisted extraction of polysaccharides from Artemisia selengensis Turcz and its antioxidant and anticancer activities. J Food Sci Technol. 2016;53 (2):1025-1034. doi:10.1007/s13197-015-2156-x

26. Luo D, Wang Z, Zhou R, et al. A polysaccharide from Umbilicaria yunnana: structural characterization and anti-inflammation effects. Int $J$ Biol Macromol. 2020;151:870-877. doi:10.1016/j. ijbiomac.2020.02.195

27. Ding J, Jin W, Chen C, et al. Tumor associated macrophage x cancer cell hybrids may acquire cancer stem cell properties in breast cancer PLoS One. 2012;7(7):e41942. doi:10.1371/journal.pone.0041942

28. Sukumar M, Roychoudhuri R, Restifo NP. Nutrient competition: a new axis of tumor immunosuppression. Cell. 2015;162 (6):1206-1208. doi:10.1016/j.cell.2015.08.064

29. Llanos S, Serrano M. Senescence and cancer: in the name of immunosuppression. Cancer Cell. 2016;30(4):507-508. doi:10.1016/j.ccell.2016.09.015

30. Wu X, Shou Q, Chen C, et al. An herbal formula attenuates collagen-induced arthritis via inhibition of JAK2-STAT3 signaling and regulation of Th17 cells in mice. Oncotarget. 2017;8 (27):44242-44254. doi:10.18632/oncotarget.17797

31. Chun JM, Kim HS, Lee AY, et al. Anti-inflammatory and antiosteoarthritis effects of saposhnikovia divaricata ethanol extract: in vitro and in vivo studies. Evid Based Complement Alternat Med. 2016;2016:1984238. doi:10.1155/2016/1984238
32. Maruyama T, Ezaki M, Shiba M, et al. Botanical origin and chemical constituents of commercial Saposhnikoviae radix and its related crude drugs available in Shaanxi and the surrounding regions. $J$ Nat Med. 2018;72(1):267-273. doi:10.1007/s11418-017-1149-7

33. Yokosuka A, Tatsuno S, Komine T, et al. Chemical constituents of the roots and rhizomes of Saposhnikovia divaricata and their cytotoxic activity. Nat Prod Commun. 2017;12(2):255-258.

34. Urbagarova BM, Shults EE, Taraskin VV, et al. Chromones and coumarins from Saposhnikovia divaricata (Turcz.) schischk. Growing in Buryatia and Mongolia and their cytotoxicity. J Ethnopharmacol. 2020;261:112517. doi:10.1016/j.jep.2019.112517

35. Kamino T, Shimokura T, Morita Y, et al. Comparative analysis of the constituents in saposhnikoviae radix and glehniae radix cum rhizoma by monitoring inhibitory activity of nitric oxide production. $J$ Nat Med. 2016;70(2):253-259. doi:10.1007/s11418-016-0969-1

36. Zhai L, Shi J, Xu W, et al. Ex vivo and in situ evaluation of 'dispelling-wind' Chinese medicine herb-drugs on intestinal absorption of chlorogenic acid. Phytother Res. 2015;29(12):1974-1981. doi:10.1002/ptr.5492

37. Nikles S, Monschein M, Zou H, et al. Metabolic profiling of the traditional Chinese medicine formulation Yu Ping Feng San for the identification of constituents relevant for effects on expression of TNF-alpha, IFN-gamma, IL-1beta and IL-4 in U937 cells. J Pharm Biomed Anal. 2017;145:219-229. doi:10.1016/j.jpba.2017.03.049

38. Batsukh Z, Toume K, Javzan B, et al. Metabolomic profiling of Saposhnikoviae radix from Mongolia by LC-IT-TOF-MS/MS and multivariate statistical analysis. J Nat Med. 2020;74(1):170-188. doi:10.1007/s11418-019-01361-0

39. Chen L, Chen X, Su L, et al. Rapid characterisation and identification of compounds in Saposhnikoviae radix by high-performance liquid chromatography coupled with electrospray ionisation quadrupole time-of-flight mass spectrometry. Nat Prod Res. 2018;32 (8):898-901. doi:10.1080/14786419.2017.1366482
OncoTargets and Therapy

\section{Publish your work in this journal}

OncoTargets and Therapy is an international, peer-reviewed, open access journal focusing on the pathological basis of all cancers, potential targets for therapy and treatment protocols employed to improve the management of cancer patients. The journal also focuses on the impact of management programs and new therapeutic agents and protocols on patient perspectives such as quality of life, adherence and satisfaction. The manuscript management system is completely online and includes a very quick and fair peer-review system, which is all easy to use. Visit http://www.dovepress.com/ testimonials.php to read real quotes from published authors. 